\title{
Tratamiento de la Enfermedad de Dupuytren en España
}

\section{Dupuytren Disease Treatment in Spain}

\author{
Rafael Sanjuan Cerveró ${ }^{1}$ Diego Gómez Herrero ${ }^{2,3}$ Carlos Novoa Parra ${ }^{1}$ Fernando Peña Molina ${ }^{1}$ \\ Nuria Franco Ferrando ${ }^{1}$ \\ 1 Servicio de Cirugía Ortopédica y Traumatología, Hospital de Denia, \\ Alicante, Spain \\ ${ }^{2}$ Servicio de Farmacia, Vithas Hospital 9 de Octubre, Valencia, Spain \\ 3 Programa de Doctorado en Farmacia, Universidad de Granada, \\ Granada, Spain \\ Address for correspondencia Rafael Sanjuan-Cerveró, MS, PhD, \\ Servicio de Cirugía Ortopédica y Traumatología, Hospital de Denia, \\ Partida Beniadlá s/n, 03700, Denia, Alicante, Spain \\ (e-mail: sanjuan.rafcer@gmail.com). \\ Rev Iberam Cir Mano 2019;47:105-114.
}

\section{Resumen \\ Palabras clave \\ - enfermedad de Dupuytren \\ - fasciectomía \\ - colagenasa \\ - encuesta}

Abstract
Keywords
- Dupuytren's disease
- fasciectomy
- collagenase
- survey

Objetivos Nuestro objetivo consiste en valorar la situación actual en España del tratamiento de la enfermedad de Dupuytren mediante una encuesta realizada a los cirujanos de mano españoles y compararla con una encuesta similar realizada en España antes de la comercialización de la colagenasa en Europa.

Material y Métodos Se remite una encuesta a los socios de la Sociedad Española de Cirugía de la Mano (SECMA) con 30 preguntas divididas en 4 bloques: aspectos demográficos, opinión general del tratamiento de la enfermedad de Dupuytren, casos clínicos y repercusión de la introducción de la colagenasa Clostridium Histolyticum en el tratamiento.

Resultados La tasa de respuesta fue del $38,2 \%$ sobre un total de 332 socios. La mayor parte de los participantes se consideró como cirujanos no noveles. La fasciectomía parcial es la técnica más frecuentemente utilizada (73\% de los encuestados) y considerada de primera elección $(61,9 \%$ ) seguida por la colagenasa $(23 \%$ y $31 \%$ respectivamente). Más de la mitad de los encuestados consideró que ha habido un cambio en el modo de afrontar la enfermedad de Dupuytren en los últimos 5 años. Con respecto a la colagenasa, actualmente el uso de los protocolos iniciales se ha abandonado, aunque el protocolo de tratamiento más habitual sigue siendo la administración de una dosis desechando el resto del vial.

Conclusiones Actualmente la fasciectomía parcial es la técnica preferida y más frecuentemente utilizada en la enfermedad de Dupuytren seguida de la colagenasa en España.

Objectives Our objective is to assess the current situation in Spain of Dupuytren's disease treatment through a survey of Spanish hand surgeons and compare it with a similar survey conducted in Spain before the commercialization of collagenase in Europe.

Methods A survey was sent to the members of the Spanish Society of Hand Surgery (SECMA) with 30 questions divided into four blocks: demographics, general opinion of Dupuytren's disease treatment, clinical reports and impact of the introduction of collagenase Clostridium Histolyticum as treatment for this pathology. received

September 15, 2019

accepted

September 24, 2019
DOI https://doi.org/

10.1055/s-0039-3399514. ISSN $1698-8396$.
Copyright $\odot 2019$ Thieme Revinter

Publicações Ltda, Rio de Janeiro, Brazil
License terms

(ㄷ) (i) $\ominus$ (5) 
Results The response rate was $38.2 \%$ over a total of 332 members. The major part of the participants were considered as non-novel surgeons. Partial fasciectomy is the most frequently used technique ( $73 \%$ of respondents) and considered first choice treatment (61.9\%) followed by collagenase ( $23 \%$ and $31 \%$ respectively). More than half of the respondents considered that there has been a change in how to deal with Dupuytren's disease in the last 5 years. With regarding collagenase, currently the use of the initial protocols has been abandoned, although the most common treatment protocol remains the administration of a dose discarding the rest of the bottle.

Conclusions Currently, partial fasciectomy is the preferred technique and more frequently used in Dupuytren's disease followed by collagenase in Spain.

\section{Introduccion}

La introducción de nuevas terapias en medicina permite nuevos abordajes terapéuticos buscando siempre el mejor beneficio del paciente, el médico y el sistema sanitario. En el caso de la enfermedad de Dupuytren (ED) la búsqueda de un tratamiento farmacológico ha sido un reto desde hace años hasta tal punto que, en 1992, John Hueston parafraseó a Martin Luther King con su famosa frase “ ¡Yo también tengo un sueño! - ¡Que un día, la enfermedad de Dupuytren será tratada sin cirugía!"1. La aparición del ensayo clínico publicado por Larry Hurst en $2009^{2}$ permitió abrir esa posibilidad con la inyección de la Colagenasa Clostridium HIstolitycum (CCH) de modo intralesional, provocando la degradación enzimática de la cuerda por parte de la enzima y evitando con ello un tratamiento quirúrgico. Desde la introducción en Europa de ese tratamiento numerosas publicaciones han demostrado su eficacia ${ }^{3}$ a corto $y$ medio plazo.

En 2011, previo a la introducción de la CCH en el mercado europeo, la Sociedad Española de Cirugía de la Mano (SECMA) auspició una encuesta para la valoración de la situación del tratamiento de la ED en España, arrojando una clara tendencia de los cirujanos españoles para con el uso de la fasciectomía parcial (FSC) como tratamiento de elección en casi el $85 \%$ de los casos, seguido con mucha diferencia por la dermofasciectomía (DFSC). Nuestro objetivo es valorar, mediante una encuesta similar promocionada a través de la SECMA y adaptada a las soluciones terapéuticas de hoy en día, la tendencia de los cirujanos españoles en el tratamiento de la ED, Así como, comprar la situación actual con la de 2011 y ver el posible impacto del tratamiento con $\mathrm{CCH}$ en nuestro entorno.

\section{Material y Metodos}

\section{Reclutamiento}

Este estudio ha contado con la aprobación por parte de la SECMA y está enmarcado dentro del proyecto SPAINCOL para la valoración del tratamiento con $\mathrm{CCH}$ a largo plazo. Ha sido aprobado por un Comité Ético Institucional (código CEI m38/ 19) y la Asociación Española de Medicamentos y Productos Sanitarios (código AEMPS RSC-COL-2019-01).
La encuesta fue realizada por el autor principal, consensuada y aprobada por el resto de autores y validada por el Comité de Investigación de la SECMA. Se probó en 2 miembros del personal médico del hospital del autor principal; sus respuestas no se incluyeron en los resultados. Tras eso, se remitió por correo electrónico a los socios de la SECMA. En la primera página se realizó una breve descripción de la encuesta, los detalles sobre el anonimato y la intención de publicar los datos identificados.

\section{Instrumento}

La encuesta se estructuró en 30 preguntas de respuesta múltiple, la mayoría de ellas con respuesta cerrada (-Supplementary Appéndix 1), diferenciándose 4 partes según contenido, que valoraban:

- Bloque 1: Preguntas 1- 4 con los aspectos demográficos de los participantes.

- Bloque 2: Preguntas 5,6,9-12,21-23 y 25-27 acerca de la opinión general del tratamiento de la ED.

- Bloque 3: Preguntas 13-20 constituidas por casos clínicos, los 4 primeros con afectación primaria y los segundos 4 casos clínicos con afectación recurrente. Este grupo de preguntas no se analizará en este artículo.

- Bloque 4: Preguntas 7-8,23-24 que evalúan la repercusión de la introducción de la $\mathrm{CCH}$ como tratamiento para la ED. Las preguntas 28-30 se realizaron con campo restringido condicionadas por la respuesta afirmativa de la pregunta número 6 , por lo que el número de participantes que contestaron esa parte del cuestionario fue menor.

Las preguntas básicas de tratamiento general y los casos clínicos $(5,9,13-20,22,25,26)$ presentaron una opción de múltiple respuesta igual para todas ellas, con 5 respuestas posibles y excluyentes (sólo se podía elegir una opción), siendo la última de ellas de campo abierto y que se analizó por separado pero que se consideró como única para el análisis estadístico. Las preguntas $7-8,10,12,27$ y 30 eran de respuesta múltiple si lo deseaba el entrevistado.

Todos los datos se muestran en base a una estadística meramente descriptiva. Las medias y las desviaciones estándar (DE) se utilizaron para las variables continuas y los porcentajes para las variables categóricas. 


\section{Resultados}

\section{Muestra}

Se remitió la encuesta a un total de 332 socios de la Sociedad. De ellos, 127 (38,2\%) respondieron la encuesta. El número de participantes fue disminuyendo conforme el número de la pregunta avanzaba de modo que en la pregunta número uno la muestra fue de 127 participantes y en la 27, de 107. En lo sucesivo se especificará en el texto el porcentaje (\%) de participantes referido a un supuesto, indicando en las tablas correspondientes el número absoluto y relativo referente a la pregunta en cuestión.

\section{Datos Demográficos}

La encuesta fue respondida en un $65,1 \%$ por cirujanos mayores de 40 años. La mayor parte de los participantes se podían considerar como cirujanos no noveles ya que el 33,9\% hacia más de 6 años que había acabado la residencia y el 45,7\% más de 15 años. Del mismo modo la mayor parte de los encuestados consistía en cirujanos de mano con experiencia (el 63,8\% de ellos tenía más de seis años de experiencia en cirugía de mano) (-Tabla $\mathbf{1}$ ).

La encuesta fue ampliamente aceptada en todo el territorio nacional. En el figura 1 se puede observar la distribución por Comunidades Autónomas. Dos participantes indicaron que trabajaban fuera de España.

\section{Tratamiento de la Enfermedad de Dupuytren}

La técnica más utilizada hoy en día y la preferida por los cirujanos de mano españoles sigue siendo la FSC (el 73\% la usan como técnica más frecuente y el 61,9\% la consideran como la técnica de primera elección), seguida por la $\mathrm{CCH}(23 \%$ y el 31\% respectivamente) (-Tabla 2 ).

La CCH ocupa un lugar en el arsenal terapéutico por sí misma en la ED y el 62,7\% de los cirujanos reconocen tener experiencia en su uso, reconociendo la mitad de los encuestados $(50,4 \%)$ un cambio de estrategia en su planteamiento acerca de la ED en los últimos 5 años. Si en este apartado eliminamos los cirujanos con menos de cinco años de experiencia en el tratamiento de la ED este porcentaje de cirujanos que considera que, personalmente, han cambiado sus estrategias de tratamiento en la ED asciende al 56,25\%. El modo en este cambio pasa por un claro aumento en las técnicas mínimamente invasivas (un $83,2 \%$ opina de esta manera, siendo un $67 \%$ a expensas de la $\mathrm{CCH}$ y un $16 \%$ a expensas de la fasciotomía percutánea) y un

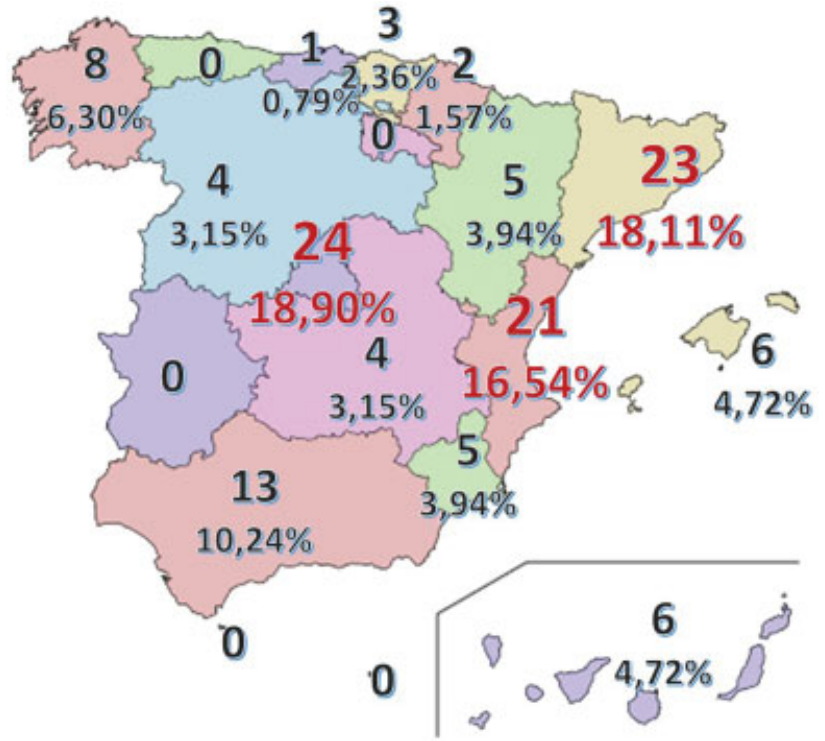

Fig. 1 Distribución geográfica de los participantes en la encuesta.

descenso en las técnicas más radicales como la DFSC (un $35,2 \%$ ). En este apartado un $8 \%$ de los encuestados respondió en campo libre otras alternativas, destacando sólo un encuestado que indicó que, según él, actualmente no se ha cambiado el modo de afrontar la ED.

En casos complejos primarios, prácticamente todos los encuestados optan por la realización de Z plastias $(94,4 \%)$ y/o artrólisis (81,6\%), siendo menos frecuentes la realización por parte de los participantes las técnicas de palma abierta (46,4\%), colgajos pediculados (20\%), amputaciones (16,8\%) o colgajos libres (12\%). Como respuestas anecdóticas 3 cirujanos especificaron en el campo de respuesta libre el empleo de artrodesis, uno la artrodesis-resección de la IFP, otro la DFSC y otro el empleo de técnica WALANT como anestesia.

En el apartado de las recurrencias ( - Tabla 3), el factor más importante para la decisión o no del tratamiento de cara a un paciente es la repercusión funcional que ocasiona la flexión del dedo (47,7\%), seguido por el grado de contractura y el estado de la piel de la zona afecta (ambas un 21,5\%). La presencia de cuerda tendinosa palpable ocupa un 9,3\% de las respuestas posiblemente al interpretarse la presencia de cuerda como factor para establecer un tratamiento y el resto de factores para planificarlo. El tratamiento más utilizado en este apartado sigue siendo la FSC $(82,2 \%)$ con

Tabla 1 Factores demográficos de los participantes $(N=127)$

\begin{tabular}{|l|l|l|l|l|l|l|l|l|}
\hline & $<30$ años & $\%$ & $30-40$ años & $\%$ & $40-50$ años & $\%$ & $>50$ años & $\%$ \\
\hline Edad de los participantes (4) & 0 & 0 & 43 & 34,5 & 38 & 30,2 & 44 & 34,9 \\
\hline & $<3$ & $\%$ & $3-6$ & $\%$ & $6-15$ & $\%$ & $>15$ & $\%$ \\
\hline $\begin{array}{l}\text { Años como desde el fin de la } \\
\text { residencia (1) }\end{array}$ & 10 & 7,9 & 16 & 12,6 & 43 & 33,9 & 58 & 45,7 \\
\hline Años como cirujano de mano * (2) & 12 & 9,4 & 22 & 17,3 & 47 & 37,0 & 34 & 26,8 \\
\hline
\end{tabular}

* Soy Cirujano Ortopédico/Plástico generalista, y dedico el mismo tiempo a valorar la patología de mano que el resto de patologías de la especialidad $N=12(9,4 \%)$. 
Tabla 2 Tratamiento general de la ED

\begin{tabular}{|c|c|c|c|c|c|c|c|c|c|c|c|}
\hline & $\mathrm{N}$ & $\mathrm{CCH}$ & $\%$ & FSC & $\%$ & DFSC & $\%$ & AA & $\%$ & Otros & $\%$ \\
\hline $\begin{array}{l}\text { Tratamiento utilizado con más } \\
\text { frecuencia (5) }\end{array}$ & 126 & 29 & 23,0 & 92 & 73,0 & 1 & 0,8 & 3 & 2,4 & $1^{\alpha}$ & 0,8 \\
\hline Tratamiento preferido (9) & 126 & 39 & 31,0 & 78 & 61,9 & 1 & 0,8 & 5 & 4,0 & $3^{\alpha}$ & 2,4 \\
\hline Tratamiento en recidivas (22) & 107 & 5 & 4,7 & 88 & 88,2 & 10 & 9,3 & 1 & 0,9 & $3^{\beta}$ & 2,80 \\
\hline Satisfacción pacientes a corto plazo ${ }^{\mu}(25)$ & 107 & 60 & 56,1 & 34 & 31,8 & 0 & 0,0 & 9 & 8,4 & 4 & 3,7 \\
\hline \multirow[t]{2}{*}{ Satisfacción pacientes a largo plazo ${ }^{\mu}(26)$} & 107 & 29 & 27,1 & 71 & 66,4 & 2 & 1,9 & 1 & 0,9 & $4^{\alpha}$ & 3,7 \\
\hline & & \multicolumn{3}{|l|}{ Sí } & \multicolumn{2}{|l|}{$\%$} & \multicolumn{3}{|l|}{ No } & \multicolumn{2}{|l|}{$\%$} \\
\hline \multirow{2}{*}{$\begin{array}{l}\text { Tiene experiencia en el uso de } \\
\mathrm{CCH} \text { para el tto. de la ED (6) }\end{array}$} & 126 & \multicolumn{3}{|l|}{79} & \multicolumn{2}{|l|}{62,7} & \multicolumn{3}{|l|}{47} & \multicolumn{2}{|l|}{37,3} \\
\hline & & Sí & \multicolumn{2}{|l|}{$\%$} & No & \multicolumn{2}{|l|}{$\%$} & \multicolumn{2}{|c|}{$<5$ a. exp } & \multicolumn{2}{|l|}{$\%$} \\
\hline \multirow{2}{*}{$\begin{array}{l}\text { ¿Ha cambiado su estrategia de afrontar la } \\
\text { ED en los últimos } 5 \text { años? (11) }\end{array}$} & 125 & 63 & \multicolumn{2}{|l|}{50,4} & 49 & \multicolumn{2}{|l|}{39,2} & \multicolumn{2}{|l|}{13} & \multicolumn{2}{|l|}{10,4} \\
\hline & & $\uparrow \mathrm{CCH}$ & $\%$ & \multicolumn{2}{|c|}{$\uparrow$ FSC } & $\%$ & \multicolumn{2}{|c|}{$\begin{array}{l}\downarrow \text { Qx } \\
\text { Radical }\end{array}$} & $\%$ & Otros & $\%$ \\
\hline Cambio en el modo de afrontar la ED (12) & 125 & 84 & 67,2 & \multicolumn{2}{|l|}{20} & 16 & \multicolumn{2}{|l|}{44} & 35,2 & 10 & 8 \\
\hline
\end{tabular}

$\alpha$-. La principal respuesta en campo abierto en la opción otros fue la técnica de fasciotomía abierta (Técnica descrita por Moermans ${ }^{28}$ ).

$\beta$-. Como respuestas en campo libre se indicaron la prótesis de Swanson ( 1 respuesta) y la artrodesis (1 caso). El caso restante dejo el campo libre sin respuesta.

$\mu-. N=107$.

Tabla 3 Tratamiento de las recurrencias $(N=107)$

\begin{tabular}{|l|l|l|l|l|l|}
\hline $\begin{array}{l}\text { Parámetro más } \\
\text { importante }\end{array}$ & N & $\%$ & $\begin{array}{l}\text { Técnica } \\
\text { empleada }\end{array}$ & N & $\%$ \\
\hline Grado contractura & 23 & 21,5 & FSC & 88 & 82,2 \\
\hline Funcionalidad & 51 & 47,7 & DFSC & 8 & 7,5 \\
\hline Cuerda palpable & 10 & 9,3 & CCH & 5 & 4,7 \\
\hline Problemas cutáneos & 23 & 21,5 & Otra & 5 & 4,7 \\
\hline
\end{tabular}

mucha diferencia, seguido por la DFSC (7,5\%) y apareciendo ya como alternativa en el tratamiento de las recidivas la administración de $\mathrm{CCH}(4,7 \%)$.

Finalmente en este apartado de la encuesta se valoraba la satisfacción de los pacientes percibida por los cirujanos (-Tabla 2). A corto plazo, la técnica que subjetivamente proporciona más satisfacción a los pacientes es la $\mathrm{CCH}$ $(56,1 \%)$ seguido de la FSC en un $31,8 \%$ e invirtiéndose prácticamente los porcentajes (27,1\% para la $\mathrm{CCH}$ y $66,4 \%$ para la FSC) en el caso de la satisfacción a largo plazo.

Como último punto se hizo referencia al método utilizado para la valoración de resultados por parte de los cirujanos, no especificándose si de modo objetivo o subjetivo. El 65,4\% de los encuestados utilizaba como método preferido la satisfacción subjetiva del paciente, mientras que solo el 30\% empleaba cuestionarios estandarizados como el DASH o el URAM.

\section{Influencia Actual del Tratamiento con CCH}

Sin duda, el factor determinante para la realización de esta encuesta, ha sido valorar la influencia que ha tenido la $\mathrm{CCH}$ en el espectro del tratamiento de la ED. Ya hemos visto como la $\mathrm{CCH}$ es la segunda técnica actualmente tanto en uso como en preferencia por los cirujanos españoles y que más de la mitad de ellos consideran que ha habido un cambio en el modo de afrontar la ED desde la introducción en el mercado de la $\mathrm{CCH}$.

Las ventajas y desventajas del tratamiento con $\mathrm{CCH}$ son variadas y la percepción del entorno laboral de cada uno puede haber influido en cada una de las respuestas (-Tabla 4). Entre las ventajas destaca la rápida reincorporación laboral del paciente $(32,5 \%)$, aunque un $35,7 \%$ respondió afirmativamente a todos los ítems y un $20 \%$ indicó que el tratamiento con $\mathrm{CCH}$ no representa ninguna ventaja sobre las respuestas expuestas. En cuanto a los inconvenientes los resultados fueron similares, ya que en el $30,2 \%$ de los casos los encuestados respondieron que todas las representadas eran inconvenientes con el tratamiento con $\mathrm{CCH}$. La opinión subjetiva de los cirujanos acerca del tratamiento con $\mathrm{CCH}$ pasa por un 58,9\% que lo considera satisfactorio y un $27,1 \%$ lo considera como útil, aunque no lo utiliza; es de destacar que un encuestado lo considera completamente insatisfactorio.

La pregunta 23 hacía referencia a la percepción de la anatomía quirúrgica tras una recidiva con un tratamiento inicial con $\mathrm{CCH}$. El 35,5\% de los encuestados reportaron que no habían tratado a un paciente de esas características y del resto de participantes $(N=69)$ el $50,7 \%$ consideraron la anatomía quirúrgica como variable, el $27,5 \%$ alterada de algún modo y el 21,7\% completamente normal. Ningún cirujano la consideró como completamente anormal.

Las tres últimas preguntas de la encuesta hacían referencia a aquellos cirujanos con experiencia en el uso con CCH ( - Tabla 5). La primera de ellas hacía referencia al mantenimiento o no de los protocolos iniciales de tratamiento con $\mathrm{CCH}$, siendo una respuesta mayoritaria su abandono actual (89,7\%) aunque 
Tabla 4 Repercusión actual del tratamiento con $\mathrm{CCH}$

\begin{tabular}{|c|c|c|}
\hline & $\mathrm{N}$ & $\%$ \\
\hline 7-. Ventajas del tratamiento con $\mathrm{CCH}$ & 126 & \\
\hline $\begin{array}{l}\text { - Rápida reincorporación a las actividades } \\
\text { de la vida diaria }\end{array}$ & 41 & 32,5 \\
\hline - Comodidad técnica para el cirujano & 26 & 20,6 \\
\hline - Pocas complicaciones terapéuticas & 28 & 14,3 \\
\hline - Coste efectividad del tratamiento & 13 & 10,3 \\
\hline - Todas & 45 & 35,7 \\
\hline - Ninguna & 25 & 19,8 \\
\hline 8-. Desventajas del tratamiento con $\mathrm{CCH}$ & 126 & \\
\hline - Tasa de recurrencia & 23 & 18,3 \\
\hline $\begin{array}{l}\text { - Necesidad de más de una dosis en } \\
\text { cuerdas o dedos múltiples }\end{array}$ & 29 & 23 \\
\hline $\begin{array}{l}\text { - Imposibilidad facturación en } \\
\text { algunos centros }\end{array}$ & 18 & 14,3 \\
\hline $\begin{array}{l}\text { - Necesidad de realizar dos citas para el } \\
\text { paciente por proceso }\end{array}$ & 6 & 4,8 \\
\hline - Todas & 38 & 30,2 \\
\hline - Ninguna & 12 & 9,5 \\
\hline $\begin{array}{l}\text { 23-. Anatomía quirúrgica tras una recidiva } \\
\text { con tratamiento } \mathrm{CCH} \text { inicial }\end{array}$ & 107 & \\
\hline - Normal & 15 & 14 \\
\hline - Alterada siendo más compleja la cirugía & 19 & 17,8 \\
\hline $\begin{array}{l}\text { - Completamente anormal, sin parecido } \\
\text { a cuerda típica }\end{array}$ & 0 & 0 \\
\hline $\begin{array}{l}\text { - Variable, aunque sin las características } \\
\text { habituales }\end{array}$ & 35 & 32,7 \\
\hline - No he intervenido ningún caso & 38 & 35,5 \\
\hline $\begin{array}{l}\text { 24-. Opinión subjetiva del tratamiento } \\
\text { con } \mathrm{CCH}\end{array}$ & 107 & \\
\hline $\begin{array}{l}\text { - Satisfactorio, lo he incorporado } \\
\text { a mi práctica clínica }\end{array}$ & 37 & 34,6 \\
\hline $\begin{array}{l}\text { - Satisfactorio, pero mayormente } \\
\text { uso otra técnica }\end{array}$ & 26 & 24,3 \\
\hline - Tiene su indicación, pero no lo uso & 29 & 27,1 \\
\hline - No creo en las ventajas de la CCH & 14 & 13,1 \\
\hline $\begin{array}{l}\text { - Completamente insatisfactorio, } \\
\text { debería obviarse }\end{array}$ & 1 & 0,9 \\
\hline
\end{tabular}

inicialmente un $47,1 \%$ de los encuestados los utilizaran. En cuanto a las complicaciones consideradas por los cirujanos como graves el $86,6 \%$ niegan haber tenido ninguna en su práctica clínica diaria, reportándose un caso de eritema localizado, un hematoma grave, un caso de adenopatías, una rotura tendinosa, un vasospasmo, una rotura cutánea de gran tamaño, una neuritis de un nervio colateral y una necrosis cutánea.

La última pregunta de la encuesta se refería a la dosis empleada en el tratamiento con CCH. Más de la mitad de los participantes $(57,4 \%)$ declararon utilizar la dosis habitual de 0,58 mg de $\mathrm{CCH}$ desechando el resto, y el 23,5\% declararon
Tabla 5 Situación actual del tratamiento con CCH en España $(N=68)$

\begin{tabular}{|c|c|c|}
\hline $\begin{array}{l}\text { 28-. Utilización de los protocolos } \\
\text { CORD* actualmente }\end{array}$ & $\mathrm{N}$ & $\%$ \\
\hline $\begin{array}{l}\text { - Uso del protocolo CORD inicialmente, } \\
\text { pero no actualmente }\end{array}$ & 32 & 47,1 \\
\hline $\begin{array}{l}\text { - Actualmente mantiene el protocolo } \\
\text { CORD para el tratamiento }\end{array}$ & 7 & 10,3 \\
\hline - Nunca he seguido los protocolos CORD & 29 & 42,6 \\
\hline \multicolumn{3}{|l|}{$\begin{array}{l}\text { 29-. ¿Ha tenido complicaciones consideradas } \\
\text { por usted como graves? }\end{array}$} \\
\hline - No & 59 & 86,8 \\
\hline - Sí & 9 & 13,2 \\
\hline \multicolumn{3}{|l|}{$\begin{array}{l}\text { 30-. Realiza algún tipo de protocolo con } \\
\text { aumento de dosis en el tratamiento }\end{array}$} \\
\hline $\begin{array}{l}\text { - No. Siempre uso la dosis habitual y } \\
\text { desecho el resto del vial }\end{array}$ & 39 & 57,4 \\
\hline - Utilizo todo el vial & 16 & 23,5 \\
\hline $\begin{array}{l}\text { - Ocasionalmente he utilizado } 2 \text { dosis en la } \\
\text { misma mano o dedo }\end{array}$ & 18 & 26,5 \\
\hline $\begin{array}{l}\text { - Ocasionalmente he utilizado } 2 \text { dosis en } \\
\text { ambas manos en un tiempo }\end{array}$ & 2 & 2,9 \\
\hline
\end{tabular}

* CORD (Collagenase Option for the Reduction of Dupuytreńs). ${ }^{2,29}$

utilizar todo el vial. El 26,5\% habían realizado infiltraciones múltiples en una misma mano en un tiempo y sólo el 2,9\% había realizado infiltraciones en las dos manos en un mismo tiempo.

\section{Discusion}

Los resultados de esta encuesta constatan claramente que la FSC es la técnica preferida y más utilizada por los cirujanos de mano españoles hoy en día al igual que en la encuesta realizada por nuestra sociedad en el año 2011.4 Sin embargo, se objetiva un claro aumento de las técnicas mínimamente invasivas, sobre todo a expensas del uso de la $\mathrm{CCH}$ y del relativo abandono de técnicas más agresivas como la DFSC incluso para casos de recurrencias.

Factores tanto personales como laborales con la rápida reincorporación a las actividades básicas de la vida diaria o las actividades profesionales, ${ }^{5}$ clínicos como la poca incidencia de complicaciones graves, ${ }^{6}$ o incluso económicos, ${ }^{7-9}$ pueden haber influido en esos resultados, ya que el tratamiento con CCH es más económico y coste efectivo a corto plazo que la FSC, ocasionando que la $\mathrm{CCH}$ vaya ocupando un lugar en el arsenal terapéutico de primera línea de la ED. Podemos considerar lejana la afirmación de Warwick ${ }^{10}$ que presuponía el uso de la $\mathrm{CCH}$ como una moda que iba a seguir un fenómeno de parábola de $S \operatorname{cott}^{11,12}$ en el que el uso, tras una moda inicial, iría desapareciendo progresivamente. De hecho, los resultados de nuestra encuesta se ven corroborados por las ventas en España del fármaco como podemos ver en la figura 2, con un aumento progresivo en el tiempo. Otros estudios objetivan 


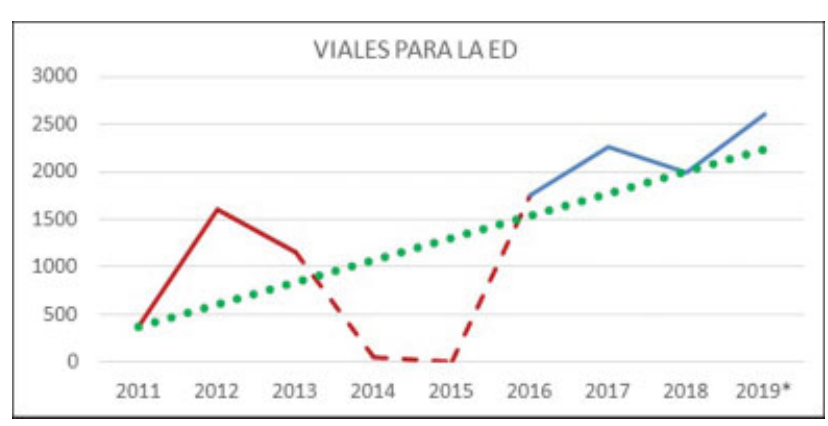

Fig. 2 Ventas de CCH para la enfermedad de Dupuytren en España. La línea roja representa la distribución por parte de Pfizer ${ }^{\circ}$ (Pfizer S.L.U., Madrid, España). La línea discontinua representa el periodo en el que Pfizer $^{\odot}$ (Pfizer S.L.U., Madrid, España) dejó la distribución hasta la comercialización por parte de Sobi ${ }^{\odot}$ (Swedish Orphan Biovitrum, S.L., Madrid, España) (línea azul). * Ventas estimadas para el 2019 relacionadas sólo con la ED. (Datos proporcionados por la industria farmacéutica).

un descenso progresivo del tratamiento de la ED mediante FSC a expensas del tratamiento con $\mathrm{CCH}^{13-15}$ con un aumento añadido de los diagnósticos de ED. ${ }^{13,15}$ Coincidimos en la opinión de esos autores, en que los pacientes antes reticentes a un tratamiento de la ED por sus consecuencias en tiempo de recuperación y la agresividad del mismo, accedan ahora a un tratamiento menos invasivo y con una recuperación más rápida. ${ }^{13,15}$

La disminución de técnicas agresivas como la DFSC, probablemente atienda a un triple factor. Por un lado la posibilidad de complicaciones graves ${ }^{16}$ a expensas de un buen resultado incluidas las rigideces en extensión tras la cirugía, una complicación grave que no suele ser recogida en los estudios clínicos. ${ }^{17}$ Por otro lado a los largos tiempos de recuperación que comprenden la cura de la herida (o el cierre por segunda intención en los casos de tratamiento a palma abierta) y la prolongada fisioterapia. ${ }^{18} \mathrm{Y}$ finalmente una tendencia actual al empleo de técnicas mínimamente invasivas pensando tanto en el deseo del paciente como en factores relacionados con una posible cirugía en el caso de recurrencias. ${ }^{19}$

Precisamente las recurrencias son el caballo de batalla en la ED. Tanto en esos casos como en los resultados de la encuesta en casos complejos iniciales, no se obvian técnicas alternativas o asociadas al proceso primario con el fin de conseguir el mejor resultado funcional, pero la cirugía de la recurrencia sigue siendo hoy en día un auténtico reto para el cirujano. ${ }^{20}$

Sin embargo, seguimos con las mismas carencias de investigación en la ED desde más de 50 años: apenas hay estudios comparativos y a largo plazo, las variaciones en la técnica quirúrgica impiden la valoración de series comparativamente, la investigación en ciencias básicas no dictamina causa, probabilidad de recurrencia o tratamiento definitivo y la valoración de resultados apenas ha tenido en cuenta la valoración del paciente. Afortunadamente parece haber una corriente de cambio y las perspectivas de futuro son prometedoras, pero seguimos sin un consenso en cuanto a los protocolos de actuación o tratamiento.
Nuestros resultados son sólo aplicables a nuestro ámbito demográfico. La distinta regulación de los sistemas sanitarios o la aprobación o no del uso de la $\mathrm{CCH}$ pueden hacer variar menormente esos resultados en distintas aéreas geográficas, como es el caso de Alemania ${ }^{21}$ donde se realizó una valoración negativa por parte de un comité federal del uso de la $\mathrm{CCH}$. Diversos estudios se han realizado tanto a lo largo de Europa ${ }^{22}$ como en $\mathrm{EEUU}^{23}$ objetivando un claro papel de la $\mathrm{CCH}$ en el arsenal terapéutico de la ED. Mcmillan ${ }^{24}$ indica una menor tendencia al tratamiento con $\mathrm{CCH}$ en los países del norte de Europa que en USA y Australia, donde, como técnica mínimamente invasiva es muy utilizada la aponeurotomía con aguja.

En cuanto a las limitaciones de nuestro estudio, pasan indudablemente por ser los resultados de una encuesta que no muestra sino las opiniones subjetivas de los participantes y que representa a una muestra de todos los cirujanos de mano de nuestro país. Del mismo modo la falta de uniformidad de las respuestas presentadas permite comparar sólo parcialmente los resultados obtenidos, no pudiéndose establecer protocolos de actuación con los resultados. También como factores confundidores podemos añadir la fatiga en la realización de la encuesta, la pérdida progresiva de participantes por esa fatiga, los sesgos producidos por las respuestas no contestadas y el error de muestra producido con el reclutamiento de los participantes.

En conclusión, podemos decir que la FSC es la técnica mayormente preferida y empleada por los cirujanos españoles. La CCH se ha establecido como un tratamiento consolidado de la ED. Sin embargo, los resultados obtenidos representan solo una muestra de los cirujanos de mano en un ámbito geográfico limitado. Una buena propuesta para la obtención de resultados concluyentes sería la realización de un estudio similar a nivel europeo tal y como se realizó hace unos años ${ }^{25-27}$ también para la ED.

\section{Conflicto de Intereses}

Los autores del trabajo declaran no tener ningún conflicto de intereses.

\section{Bibliografía}

1 Hueston J. Lessons in Dupuytren's disease. Ann Chir Main Memb Super 1992;11(05):349-354. Doi:10.1016/S0753-9053(05)80269-8

2 Hurst LC, Badalamente MA, Hentz VR, et al; CORD I Study Group. Injectable collagenase clostridium histolyticum for Dupuytren's contracture. N Engl J Med 2009;361(10):968-979. Doi:10.1056/ NEJMoa0810866

3 Sanjuan-Cerveró R, Vazquez-Ferreiro P, Gomez-Herrero D, Carrera-Hueso FJ. Efficacy of Collagenase Clostridium Histolyticum for Dupuytren Disease: A Systematic Review. Rev Iberoam Cir Mano 2017;45(02):70-88

4 Muñoz-Peñin R, Lwoff N, Renú JMA. Enfermedad de Dupuytren, una vista panorámica de nuestro entorno. Rev Iberoam Cir Mano 2011;39(02):126-134. Doi:10.1055/s-0037-1606801

5 Naam NH. Functional outcome of collagenase injections compared with fasciectomy in treatment of Dupuytren's contracture. Hand ( $\mathrm{N}$ Y) 2013;8(04):410-416. Doi:10.1007/s11552-013-9540-7

6 Sanjuan-Cerveró R, Carrera-Hueso FJ, Vazquez-Ferreiro P, GomezHerrero D. Adverse Effects of Collagenase in the Treatment of 
Dupuytren Disease: A Systematic Review. BioDrugs 2017;31(02): 105-115. Doi:10.1007/s40259-017-0211-z

7 Chen NC, Shauver MJ, Chung KC. Cost-effectiveness of open partial fasciectomy, needle aponeurotomy, and collagenase injection for dupuytren contracture. J Hand Surg Am 2011;36(11):1826-1834. e32. Doi:10.1016/j.jhsa.2011.08.004

8 Mehta S, Belcher HJCR. A single-centre cost comparison analysis of collagenase injection versus surgical fasciectomy for Dupuytren's contracture of the hand. JPlast Reconstr Aesthet Surg 2014;67(03):368-372. Doi:10.1016/j.bjps.2013.12.030

9 Sanjuan Cerveró R, Franco Ferrando N, Poquet Jornet J. Use of resources and costs associated with the treatment of Dupuytren's contracture at an orthopedics and traumatology surgery department in Denia (Spain): collagenase clostridium hystolyticum versus subtotal fasciectomy. BMC Musculoskelet Disord 2013;14(01):293. DOI: $10.1186 / 1471-2474-14-293$

10 Warwick D. Collagenase: What We May Never Know (A Discussion). In: Werker PMN, Dias J, Eaton C, Reichert B, Wach W, eds. Dupuytren Disease and Related Diseases - The Cutting Edge. Springer International Publishing; 2017:145-149. doi:10.1007/978-3-31932199-8_19

11 Jupiter J, Burke D. Scott's parabola and the rise of the medicalindustrial complex. Hand (N Y) 2013;8(03):249-252. Doi:10.1007/ s11552-013-9526-5

12 ScottJW. Scott's parabola. BMJ 2001;323(7327):1477. DOI: 10.1136/ bmj.323.7327.1477

13 Lipman MD, Carstensen SE, Deal DN. Trends in the Treatment of Dupuytren Disease in the United States Between 2007 and 2014. Hand (N Y) 2017;12(01):13-20. Doi:10.1177/1558944716647101

14 Thayer MK, Somerson JS, Huang JI. Changes in Provider Treatment Patterns for Dupuytren's Contracture: Analysis of Trends in Medicare Beneficiaries. Plast Reconstr Surg Glob Open 2018;6 (10):e1932. DOI: 10.1097/GOX.0000000000001932

15 Zhao JZ, Hadley S, Floyd E, Earp BE, Blazar PE. The Impact of Collagenase Clostridium histolyticum Introduction on Dupuytren Treatment Patterns in the United States. J Hand Surg Am 2016;41 (10):963-968. Doi:10.1016/j.jhsa.2016.07.090

16 Crean SM, Gerber RA, Le Graverand MP, Boyd DM, Cappelleri JC. The efficacy and safety of fasciectomy and fasciotomy for Dupuytren's contracture in European patients: a structured review of published studies. JHand Surg Eur Vol 2011;36(05): 396-407. Doi:10.1177/1753193410397971

17 Sennwald GR. Fasciectomy for treatment of Dupuytren's disease and early complications. JHand Surg Am 1990;15(05):755-761
18 Lesiak AC, Jarrett NJ, Imbriglia JE. Modified McCash Technique for Management of Dupuytren Contracture. J Hand Surg Am 2017;42 (05):395.e1-395.e5. Doi:10.1016/j.jhsa.2017.01.018

19 Arandes-Renú JM, Sanjuán-Cerveró R, Moya-Molinas I. Collagenase in Europe from the Experimental Phase to Practical Use. In: Pajardi G, Badalamente MA, Hurst LC, eds. Collagenase in Dupuytren Disease. Cham: Springer International Publishing; 2018:39-74. doi:10.1007/978-3-319-65822-3_4

20 Eaton C. Dupuytren and Rare Disease Research. Dupuytren Research Group. https://dupuytrens.org/dupuytren-and-raredisease-research/. Published July 1, 2019. Accessed July 30, 2019.

21 Reichert B, Baringer M. [Preferred treatment options of German hand surgeons in Dupuytren disease]. Handchir Mikrochir Plast Chir 2018;50(03):196-201. Doi:10.1055/a-0645-7075

22 Marks M, Krefter C, Herren DB. [Treatment Methods for Patients with Dupuytren's Disease in Switzerland]. Handchir Mikrochir Plast Chir 2016;48(03):155-160. Doi:10.1055/s-0042-104056

23 Carr L, Michelotti B, Brgoch M, Hauck R, Ingraham J. Dupuytren Disease Management Trends: A Survey of Hand Surgeons. Hand (N Y) 2018;1558944718787281(July):1558944718787281. DOI: 10.1177| 1558944718787281

24 McMillan C, Yeung C, Binhammer P. Variation in Treatment Recommendations for Dupuytren Disease. JHand Surg Am 2017;42(12):963-970.e6. Doi:10.1016/j.jhsa.2017.08.023

25 Bainbridge C, Dahlin LB, Szczypa PP, Cappelleri JC, Guérin D, Gerber RA. Current trends in the surgical management of Dupuytren's disease in Europe: an analysis of patient charts. Eur Orthop Traumatol 2012;3(01):31-41. Doi:10.1007/s12570-012-0092-z

26 Dahlin LB, Bainbridge C, Leclercq C, et al. Dupuytren's disease presentation, referral pathways and resource utilisation in Europe: regional analysis of a surgeon survey and patient chart review. Int J Clin Pract 2013;67(03):261-270. Doi:10.1111/ ijcp.12099

27 Dias J, Bainbridge C, Leclercq C, et al. Surgical management of Dupuytren's contracture in Europe: regional analysis of a surgeon survey and patient chart review. Int J Clin Pract 2013;67(03): 271-281. Doi:10.1111/ijcp.12106

28 Moermans JP. Segmental aponeurectomy in Dupuytren's disease. J Hand Surg [Br] 1991;16(03):243-254. Doi:10.1016/0266-7681 (91)90047-R

29 Gilpin D, Coleman S, Hall S, Houston A, Karrasch J, Jones N. Injectable collagenase Clostridium histolyticum: a new nonsurgical treatment for Dupuytren's disease. JHand Surg Am 2010;35(12):2027-38.e1. Doi:10.1016/j.jhsa.2010.08.007 


\section{Anexo 1 Encuesta remitida a los participantes.}

\section{ENCUESTA SOBRE TECNICAS DE TRATAMIENTO EN LA ENFERMEDAD DE DUPUYTREN}

1. ¿Cuántos años lleva ejerciendo su especialidad desde que acabó la residencia?

a) Menos de 3

b) De 3 a 6

c) De 6 a 15

d) Más de 15

2. ¿Y cómo cirujano dedicado la mano?
a) Menos de 3
b) De 3 a 6
c) De 6 a 15
d) Más de 15
e) Soy Cirujano Ortopédico/Plástico generalista, y dedico el mismo tiempo a valorar la patología de mano que el resto de patologías de la especialidad.

3. ¿Podría indicar la Comunidad Autónoma donde preferentemente ejerce su profesión?

4. ¿Podría indicarnos su edad?
a) Menos de 30
b) Entre 30 y 40
c) Entre 40 y 50
d) Más de 50

5. En la enfermedad de Dupuytren, ¿cuál de los siguientes tratamientos es el que utiliza con más frecuencia?

a) Inyección de colagenasa Clostridium HIstolyticum

b) Fasciectomía abierta

c) Dermofasciectomía

d) Fasciotomía percutánea con aguja

e) Otras técnicas: Indique cuales por favor:

6. ¿Tiene experiencia con la inyección de colagenasa del Clostridium Histolyticum como opción terapéutica en la enfermedad de Dupuytren?

a) No

b) $\mathrm{Si}$

7. ¿Cuál opina que es la principal ventaja de la inyección de colagenasa del Clostridium Histolyticum? Marque varias si considera oportuno

a) El coste-efectividad para el sistema sanitario

b) Las pocas complicaciones terapéuticas

c) La rápida reincorporación del paciente a sus actividades de la vida diaria

d) La comodidad de la técnica para el cirujano

e) Todas

f) Ninguna

8. ¿Y la principal desventaja?

a) La tasa de recurrencia

b) Necesidad de más de una dosis en cuerdas múltiples o varios dedos afectos c) La imposibilidad de facturación en algunos centros

d) Necesidad de citar al paciente dos días

e) Todas

f) Ninguna

9. Independientemente de la frecuencia, ¿cuál es el tratamiento preferido por usted?

a) Inyección de colagenasa Clostridium HIstolyticum

b) Fasciectomía abierta

c) Dermofasciectomía

d) Fasciotomía percutánea con aguja

e) Otras técnicas: Indique cuales por favor:

10. Antes situaciones especiales utiliza usted alguna de estas variantes. Marque varias si lo considera oportuno.
a) Z plastias
b) Técnica de palma abierta
c) Colgajos pediculados
d) Colgajos a distancia
e) Amputaciones
f) Artrolisis IFP.
g) Otras técnicas. Indique cuales:

11. ¿Considera que ha cambiado su estrategia de afrontar la enfermedad de Dupuytren en los últimos 5 años?

a) $\mathrm{Si}$

b) No

c) Llevo menos de 5 años tratando la enfermedad de Dupuytren

12. ¿Cómo considera que ha cambiado en general el modo de afrontar la enfermedad de Dupuytren? Marque varias si lo considera oportuno

a) Aumento del tratamiento con Inyección de colagenasa Clostridium HIstolyticum

b) Aumento del tratamiento con Fasciotomía percutánea

c) Disminución de las intervenciones quirúrgicas radicales

d) Otras razones:

13. En un paciente de 75 años, con una contractura de IFP del $5^{\circ}$ dedo $>70^{\circ}$, sin afectación de la MCTF, ¿cuál es el tratamiento de elección en su práctica habitual?

a) Inyección de colagenasa Clostridium HIstolyticum

b) Fasciectomía abierta

c) Dermofasciectomía

d) Fasciotomía percutánea con aguja

e) Otras técnicas: Indique cuales por favor:

14. En un paciente de 45 años, con afectación aislada del dedo central con una contractura MTCF de $40^{\circ}$, sin afectación de IFP, ¿Cuál es el tratamiento de elección en su práctica habitual?

a) Inyección de colagenasa Clostridium HIstolyticum

b) Fasciectomía abierta 
c) Dermofasciectomía

d) Fasciotomía percutánea con aguja

e) Otras técnicas: Indique cuales por favor:

15. Ante una afectación del $4^{\circ}$ y $5^{\circ}$ dedo, con angulaciones $>45^{\circ}$ en el valor conjunto de la medición de la MCTF y de la IFP (MCTF, IFP o MCTF + IFP), ¿cuál es el tratamiento de elección en su práctica habitual?

a) Inyección de colagenasa Clostridium HIstolyticum

b) Fasciectomía abierta

c) Dermofasciectomía

d) Fasciotomía percutánea con aguja

e) Otras técnicas: Indique cuales por favor:

16. En un paciente de 45 años con afectación en forma de nódulo a nivel de la $\mathrm{F} 1$ del $3^{\circ}$ dedo, con una flexión de $30^{\circ}$ de la MCTF y $15^{\circ}$ de la IFP ¿cuál seria su tratamiento de primera elección?

a) Inyección de colagenasa Clostridium HIstolyticum

b) Fasciectomía abierta

c) Dermofasciectomía

d) Fasciotomía percutánea con aguja

e) Otras técnicas: Indique cuales por favor:

17. En un paciente de 72 años, con recurrencia de una infiltración previa de inyección de colagenasa del Clostridium Histolyticum sobre el $5^{\circ}$ dedo, con una contractura de $20^{\circ}$ de la MTCF y $40^{\circ}$ de la IFP. ¿cuál sería su tratamiento de primera elección?
a) Inyección de colagenasa Clostridium HIstolyticum
b) Fasciectomía abierta
c) Dermofasciectomía
d) Fasciotomía percutánea con aguja
e) Otras técnicas: Indique cuales por favor:

18. En un paciente de 50 años, con recurrencia de una infiltración previa de inyección de colagenasa del Clostridium Histolyticum sobre el $4^{\circ}$ dedo de forma aislada y $40^{\circ}$ de contractura de la MTCF, ¿cuál sería su tratamiento de primera elección?
a) Inyección de colagenasa Clostridium HIstolyticum
b) Fasciectomía abierta
c) Dermofasciectomía
d) Fasciotomía percutánea con aguja
e) Otras técnicas: Indique cuales por favor:

19. En un paciente de 72 años, con recurrencia de una fasciectomía previa sobre el $5^{\circ}$ dedo y una contractura de $20^{\circ}$ de la MCTF y $40^{\circ}$ de la IFP, ¿cuál sería su tratamiento de primera elección?
a) Inyección de colagenasa Clostridium HIstolyticum
b) Fasciectomía abierta
c) Dermofasciectomía
d) Fasciotomía percutánea con aguja
e) Otras técnicas: Indique cuales por favor:

20. En un paciente de 50 años, con recurrencia de una fasciectomía previa sobre el $4^{\circ}$ dedo de forma aislada y $40^{\circ}$ de contractura de la MCTF, ¿cuál sería su tratamiento de primera elección?
a) Inyección de colagenasa Clostridium HIstolyticum
b) Fasciectomía abierta
c) Dermofasciectomía
d) Fasciotomía percutánea con aguja
e) Otras técnicas: Indique cuales por favor:

21. Con respecto a las recurrencias, ¿qué parámetro tiene mayor importancia para usted en la planificación de un nuevo tratamiento?
a) El grado de contractura de la zona afecta
b) La repercusión funcional en el paciente
c) La existencia de cuerda pretendinosa palpable
d) El estado de la piel por cirugías previas

22. En las recidivas de la enfermedad de Dupuytren en general, ¿cuál es el tratamiento más utilizado por usted?
a) Inyección de colagenasa Clostridium HIstolyticum
b) Fasciectomía abierta
c) Dermofasciectomía
d) Fasciotomía percutánea con aguja
e) Otras técnicas: Indique cuales por favor:

23. Si ha realizado una fasciectomía tras recidiva tras un tratamiento inicial con inyección de colagenasa del Clostridium Histolyticum, la anatomía quirúrgica la puede definir como:

a) Completamente normal

b) Alterada, siendo más complicada la cirugía

c) Completamente anormal, sin parecido a cuerda de Dupuytren típica

d) Variable según el caso, aunque con una cuerda sin las características habituales

e) No he intervenido ningún caso como el descrito

24. ¿Cuál es su opinión subjetiva acerca del tratamiento con inyección de colagenasa del Clostridium Histolyticum?

a) Satisfactorio, lo he incorporado a mi práctica habitual.

b) Satisfactorio, pero sigo utilizando mayoritariamente otras técnicas

c) Es un tratamiento que me parece tiene su indicación, pero personalmente no lo utilizo.

d) No creo en las ventajas de la inyección de colagenasa del Clostridium Histolyticum en el tratamiento de la enfermedad de Dupuytren.

e) Completamente insatisfactorio. Es un método de tratamiento que debería obviarse para la enfermedad de Dupuytren.

25. Personalmente, ¿con que técnica cree que sus pacientes se encuentran más satisfechos a corto plazo?
a) Inyección de colagenasa Clostridium HIstolyticum
b) Fasciectomía abierta
c) Dermofasciectomía
d) Fasciotomía percutánea con aguja
e) Otras técnicas: Indique cuales por favor:

26. Y ¿a largo plazo?

a) Inyección de colagenasa Clostridium HIstolyticum

b) Fasciectomía abierta 
c) Dermofasciectomía

d) Fasciotomía percutánea con aguja

e) Otras técnicas: Indique cuales por favor:

27. Para la valoración de sus resultados en el tratamiento de la enfermedad de Dupuytren, ¿qué método sigue habitualmente? (Marque varios si lo considera conveniente).

a) Valoración únicamente por la medición de la extensión obtenida en el dedo

b) Valoración únicamente por la extensión conseguida del dedo con la clasificación de Tubiana

c) Aplicación de cuestionarios específicos (DASH, QuickDASH, MHS, BriefMHS, URAM...)

d) Satisfacción subjetiva del paciente

28. Los estudios pivotales diseñados para la valoración del tratamiento con inyección de colagenasa del Clostridium Histolyticum (estudios CORD) establecían la administración del tratamiento hasta en 3 ocasiones, para alcanzar la extensión completa del dedo. Por contra diversos autores, por problemas de coste-efectividad y disponibilidad del fármaco principalmente, redujeron la administración a dosis únicas, independientemente del resultado. Atendiendo a estas premisas, ¿cuál es su postura?:

a) Utilicé inicialmente el protocolo de los estudios CORD, aunque actualmente ya no lo utilizo b) Utilicé y sigo manteniendo el protocolo establecido en los estudios CORD

c) No he seguido nunca el protocolo establecido en los estudios CORD.

29. En cuanto al tratamiento con inyección de colagenasa del Clostridium Histolyticum, ¿ha tenido alguna complicación que pueda definir usted como grave?

a) No

b) SI. Describa cuál/es:

30. Actualmente se están publicando diversos trabajos acerca de la utilización de inyección de colagenasa del Clostridium Histolyticum con una dosis superior a la habitual $(0,58 \mathrm{mg})$. ¿Podría indicar si realiza alguna variación con respecto a la administración de la dosis estándar? (Marque varias a la vez si lo considera oportuno)

a) No, siempre utilizo la dosis habitual y desecho el resto

b) Suelo administrar más dosis de la habitual, utilizando todo el vial disponible

c) En ocasiones he administrado dos dosis en la misma mano o dedo si el paciente así lo requiere para finalizar el tratamiento en un solo tiempo

d) En ocasiones he infiltrado a pacientes en las dos manos si hay afectación bilateral. 